\title{
Catching ease influences perceived speed: Evidence for action-specific effects from action-based measures
}

\author{
Jessica K. Witt • Mila Sugovic
}

Published online: 9 May 2013

(C) Psychonomic Society, Inc. 2013

\begin{abstract}
According to the action-specific account of perception, people perceive the environment in terms of their ability to act. Here, we directly tested this claim by using an action-based measure of perceived speed: Participants attempted to catch a virtual fish by releasing a virtual net. The net varied in size, making the task easier or harder. We measured perceived speed by using explicit judgment-based measures and an action-based measure (time to release the net). Participants released the net later when playing with the big as compared with the small net, indicating that the fish looked to be moving more slowly when participants played with the big net. Explicit judgments of fish speed were similarly influenced by net size. These results provide converging evidence from both explicit and action-based measures that a perceiver's ability to act influences a common underlying process, most likely perceived speed, rather than postperceptual processes such as response formation.
\end{abstract}

\section{Keywords Embodied cognition · Visual perception}

Perception evolved to provide the organism with useful information about the environment. The success of perception depends not on its geometric accuracy, but on the extent to which it leads to appropriate behavioral decisions and successful guidance of actions. A naïve and perhaps common assumption is that the best perception is the one that most closely matches geometric veridicality. Indeed, with

Electronic supplementary material The online version of this article (doi:10.3758/s13423-013-0448-6) contains supplementary material, which is available to authorized users.

\section{J. K. Witt $(\bowtie)$}

Department of Psychology, Colorado State University,

Fort Collins, CO 80523, USA

e-mail: Jessica.Witt@colostate.edu

M. Sugovic

Department of Psychological Sciences, Purdue University,

West Lafayette, IN 47907, USA respect to guiding actions, the perceptual information processed by the dorsal pathway has been recognized for its resistance to visual illusions (e.g., Ganal, Tanzer, \& Goodale, 2008).

However, with respect to behavioral decisions, the assumption regarding the benefits of geometric accuracy has recently been challenged by the action-specific account of perception. According to this account, people perceive the environment in terms of their ability to act (Proffitt, 2006; Witt, 2011a). For example, when a perceiver is fatigued, carries a heavy backpack, or is of poor physical fitness, hills look steeper (Bhalla \& Proffitt, 1999). These effects are potentially useful for making decisions about which actions to perform. Seeing a hill as being steeper might help the perceiver to select an appropriate walking pace that would help conserve energy and avoid exhaustion (Proffitt, 2006), or seeing a threatening object as being closer or faster might promote faster responses to the threat (Cole, Balcetis, \& Dunning, 2013; Witt \& Sugovic, in press). Furthermore, a hunter who sees the target as being bigger or closer when shooting well (cf. Lee, Lee, Carello, \& Turvey, 2012) might make the decision to shoot from his or her current location rather than move closer and risk being detected by the prey (Witt, 2011a).

According to the action-specific approach, the same object looks different depending on the perceiver's abilities. But rather than considering these effects as errors, the actionspecific account promotes the idea that perception expresses the relationship between the perceiver and the environment: As this relationship changes, perception also changes. Like Gibson's (1979) ecological approach, the action-specific account emphasizes the perceiver-environment relationship, rather than the idea that perception provides a behaviorally independent representation of the environment.

However, some researchers have questioned whether action-specific effects are truly perceptual. An alternative explanation is that these effects exert themselves on postperceptual processes that are involved in generating a 
response (Loomis \& Philbeck, 2008). Perception cannot be measured directly, so researchers must make inferences about perception on the basis of responses. In typical perception experiments, researchers ask participants to make judgments about, for example, target distance, size, or speed. However, there is concern about whether these types of judgments reflect perceptual experiences or postperceptual processes, such as those related to response bias and task demands (e.g., Durgin, Baird, Greenburg, Russell, Shaughnessy, \& Waymouth, 2009; Loomis \& Philbeck, 2008). Consequently, determining the locus of an effect, and whether it is perceptual, poses a challenge.

To determine whether action-specific effects reflect changes in perception, Loomis and Philbeck (2008) suggested using two techniques. The first of these was to use indirect measures. For example, perceived shape provides an indirect measure of perceived distance: If the target really looks closer, then the shape formed by the position of the target relative to other objects will look different. Using perceived shape as an indirect measure, Witt (2011b) found that modifications to a person's ability to reach influenced perceived distance. Perceived size also provides an indirect measure of perceived distance: If the target looks closer, it should also look smaller. Using perceived size as the measure, Stefanucci and Proffitt (2009) found that fear of heights influenced perceived height from atop a balcony. In both of these cases, participants made perceptual judgments, but the measures were indirect, and thus were less susceptible to response-related biases.

The second recommended technique was to use actionbased measures. If a perceiver sees an object differently, subsequent actions should reveal this perceptual bias. Action-based measures have the distinct advantage that they do not require explicit judgments. This is important because explicit judgments are specifically the ones that have led to concerns over the nature of these effects (see, e.g., Durgin et al., 2009). Previously, we used the action-based measure of blindwalking to examine the effects of effort on perceived distance (Witt, Proffitt, \& Epstein, 2010); the distance that participants blindwalked to a target was used as a measure of perceived distance. Participants who anticipated having to exert more effort blindwalked farther than did participants who anticipated having to exert less effort, suggesting that they perceived the target to be farther away.

However, blindwalking is admittedly a strange measure of perception, given that perceivers have their eyes closed while making the response. A more direct way to assess whether a person's ability to act truly influences perception would be to have participants perform an action while looking at the target. This kind of action-based measure would directly test the specific claim that a person's ability to act influences his or her perception, in comparison to the alternative explanations (Durgin et al., 2009; Loomis \&
Philbeck, 2008). Given the controversy over whether action-specific effects are perceptual, this type of actionbased measure could help determine the underlying nature of these effects.

\section{Method}

Participants

A group of 13 students participated in exchange for course credit. All were naïve to the purpose of the study, and all provided informed consent.

Stimulus and apparatus

All stimuli were displayed on a 19-in. monitor with a black background (see Fig. 1). The target object was a white fish that was $1.8 \mathrm{~cm}$ tall and $1.9 \mathrm{~cm}$ long and that always started $1 \mathrm{~cm}$ from the left edge of the monitor. During the exposure and review phases, the fish was $14.3 \mathrm{~cm}$ from the bottom of the monitor. During test trials, the fish was either high (21.4 $\mathrm{cm}$ from the bottom) or low $(17.5 \mathrm{~cm}$ from the bottom). The net was white, $0.7 \mathrm{~cm}$ wide, and was set to one of three heights $(1.7,5.6$, and $11.5 \mathrm{~cm})$ on each trial. The center of the net was initially positioned $6.5 \mathrm{~cm}$ from the bottom of the monitor and $2.4 \mathrm{~cm}$ from the right edge.

\section{Procedure}

The participants were initially trained on Speeds 1 and 7 . This training consisted of an exposure phase and a review phase. During the exposure phase, text on the screen indicated which speed would be shown; then the fish traveled left to right across the screen, with no vertical displacement, at $0.18 \mathrm{~m} / \mathrm{s}$ (Speed 1) or $0.74 \mathrm{~m} / \mathrm{s}$ (Speed 7). Each speed was repeated three times, and the order of presentation was randomized. During the review phase, no text indicated the speed. In this phase, the fish traveled at Speed 1 or 7, and participants had to indicate the speed by pressing " 1 " or "7" on the keyboard. Again, each speed was presented three times, and the order was randomized.

During the test trials, the fish moved across the screen at one of six speeds (ranging from 0.18 to $0.65 \mathrm{~m} / \mathrm{s}$ ), and the net was set to be small, medium, or big. The fish and net were displayed for $50 \mathrm{~ms}$ before the fish started to move. To release the net, participants pressed the trigger on the joystick. Upon release, the net moved upward at a constant speed $(0.19 \mathrm{~m} / \mathrm{s})$. If the net intersected the fish, this was a successful catch, and the fish stopped on the net. If the net did not intersect the fish, both the net and the fish continued beyond the edges of the screen and the trial was coded as a miss. Then participants estimated the speed of the fish on a 
Fig. 1 Example of the stimuli at the start (left) and end (right) of a trial. Here, the medium net is depicted
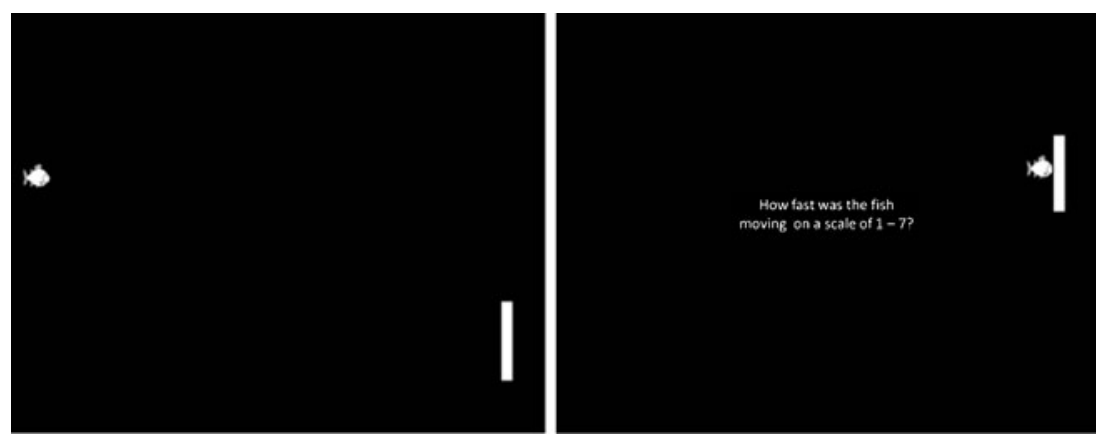

scale of $1-7$, by pressing the corresponding button on the keyboard. Each block contained 36 trials $(3$ net sizes $\times 6$ speeds $\times 2$ fish positions) whose order was randomized within a block. Participants completed one practice block and seven test blocks.

\section{Results}

One participant did not understand the task and never released the net. His data were thus removed. We first assessed whether our manipulation of ability (net size) affected performance. The mean numbers of fish successfully caught were entered into a repeated measures analysis of variance (ANOVA) with Net Size, Speed, and Vertical Fish Position as within-subjects factors. As expected, net size significantly influenced catching success, $F(2,22)>402, p<.001$, $\eta_{\mathrm{p}}{ }^{2}=.97$; participants caught more fish as net size increased. All other main effects and all interactions were significant as well, $F \mathrm{~s}>9, p \mathrm{~s}<.001, \eta_{\mathrm{p}}{ }^{2} \mathrm{~s}>.46$ (see Fig. 2).

\section{Explicit judgments}

To analyze our judgment-based measure, we entered speed ratings into a repeated measures ANOVA with Net Size, Fish Position, and Fish Speed as within-subjects factors. Net size influenced estimated speed, $F(2,20)=7.64$, $p<.01, \eta_{\mathrm{p}}{ }^{2}=.43$; participants estimated the fish to be moving faster when they played with the smaller net (see Fig. 3). Fish speed also influenced estimated speed, $F(5,50)>242, \eta_{\mathrm{p}}{ }^{2}=.99$, and fish position had a marginally significant effect on estimated speed, $F(1,10)$ $=3.50, p=.09, \eta_{\mathrm{p}}{ }^{2}=.26$ : Participants tended to estimate that the higher fish was moving faster.

In these tasks, we typically analyze how task ease-as determined by the size of the net-influences apparent speed. We can also look at how trial-by-trial performance affects perceptual judgments. With the previous Pong-like paradigm, we found that trial-by-trial performance (i.e., misses vs. hits) sometimes influenced perceptual judgments, but more often did not influence speed judgments (Witt \& Sugovic, 2010, 2012). Unlike in the Pong paradigm, with this fish paradigm, we could examine whether the kind of miss (i.e., too late vs. too early) influenced judgments.

Not all performance outcomes occurred for each speed and net size combination. For example, participants were never early for the fastest speeds, and were rarely late for the slowest speeds. For each combination, whenever the data permitted, we ran separate paired-sample $t$ tests to compare mean explicit speed judgments when participants were early versus on time and when they were on time versus late. With Bonferroni correction $(\alpha=.0025)$, none of these tests reached significance ( $t \mathrm{~s}$ ranged from -3.22 to $2.58, p \mathrm{~s}=.012-.895$ ). Thus, whereas task ease (manipulated in the form of net size)
Fig. 2 Proportions of fish caught, as a function of net size and fish position (left) and as a function of net size and fish speed (right). Error bars represent 1 SEM calculated within subjects
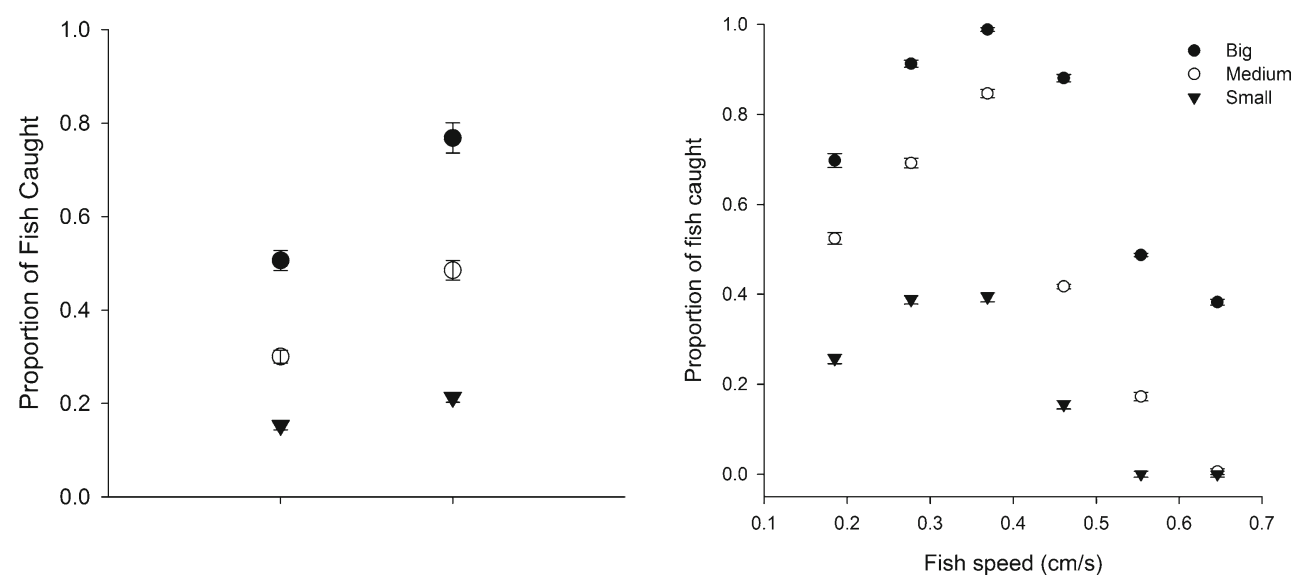


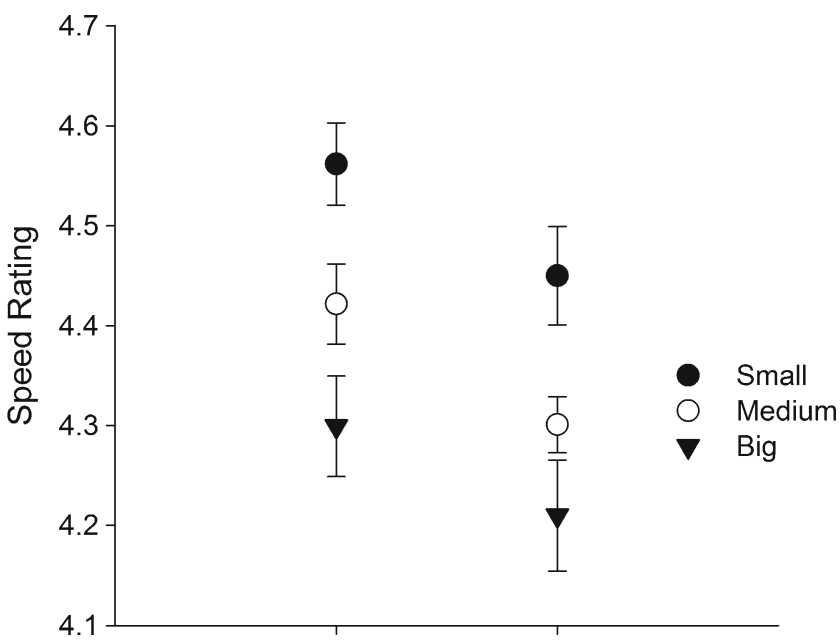

Fig. 3 Speed ratings as a function of net size and fish position. A higher value indicates seeing the fish as moving faster. Error bars represent 1 $S E M$, calculated within subjects for each fish position

influenced perceptual judgments of speed, individual trial performance did not significantly influence speed judgments. This result also shows that participants did not modify their speed judgments on the basis of whether or not they were successful on any given trial.

\section{Action-based measure}

If participants actually perceived the fish to be moving slower when the net was bigger, this should be revealed by their actions. Specifically, they should have waited longer to release the big net than to release the small net. We analyzed net release times to determine whether net size influenced this action-based measure of apparent speed. Outliers (determined as release times that were greater than 3 the interquartile range for each fish speed) were removed ( $1 \%$ of trials). We conducted a repeated measures ANOVA with Net Release Time as the dependent factor and Net Size, Fish Speed, and Fish Position as repeated factors. Net size significantly influenced net release times, $F(2,22)=4.85, p<.05, \eta_{\mathrm{p}}{ }^{2}=.31$; participants released the net later when they played with the bigger net (see Fig. 4). This pattern is indicative of seeing the fish as moving more slowly when participants were playing with the bigger net. Participants also released the net sooner when the fish was positioned higher than when it was lower, $F(1,11)>66$, and when the fish was moving faster, $F(5,55)>175$. The interaction between fish position and fish speed was also significant, $F>28$; no other interactions were significant, $p \mathrm{~s}>.24$.

A similar pattern emerged when we analyzed the data for which participants successfully caught the fish. Because participants did not successfully catch fish under all conditions, these data were analyzed with a univariate ANOVA,

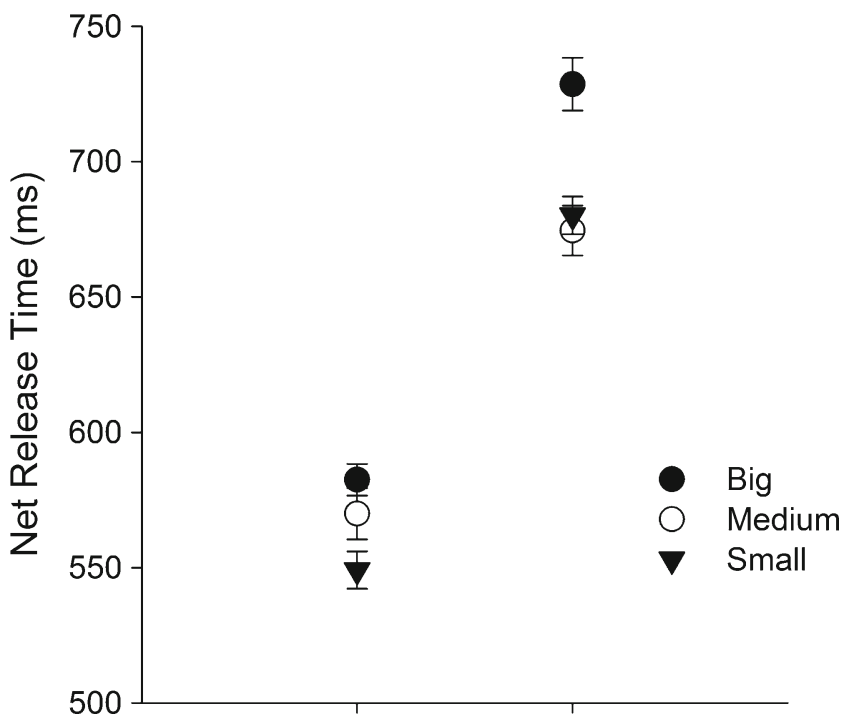

Fig. 4 Net release times as a function of net size and fish position. A lower value indicates releasing the net sooner and is indicative of seeing the fish as moving faster. Error bars represent $1 S E M$, calculated within subjects for each fish position

with Net Release Time as the dependent factor; Net Size, Fish Speed, and Fish Position as independent factors; and Participant Number as a random factor. As before, net size influenced net release time, $F(22,33.17)=56.55, p<.001$, $\eta_{\mathrm{p}}^{2}=.77$ : Even on successful trials, participants waited longer to release the big net $(M=785 \mathrm{~ms}, S E=13)$ than to release the medium $(M=657 \mathrm{~ms}, S E=17)$ or the small $(M=621 \mathrm{~ms}, S E=27)$ net. This pattern is consistent with the idea that the fish looked to be moving more slowly when participants were playing with a bigger net.

Other factors can influence net release time, so it was important to assess whether our results could be explained by other possible factors than the proposed effects on perceived speed. One possibility is that the strategy used to catch the fish influenced perceived speed. If, for example, participants had aimed to catch the fish with the top portion of the net, the big net could have been released later due to catching strategy rather than to the proposed differences in perceived speed. However, this strategy seems unlikely, as the task was difficult, and the strategy that would have maximized performance would be to aim to catch the fish at the middle of the net. Indeed, people are good at accounting for variability in their movements and aiming at the optimal location to achieve the highest rate of success (Trommershäuser, Maloney, \& Landy, 2008).

The data from net positions at the time of fish intersection also suggest that participants did not employ the strategy of catching the fish with the top of the net. When the fish moved slowly enough that participants could employ an aiming strategy (rather than just responding as quickly as 
possible), they caught the fish in the middle of the net (see the online supplementary materials). Additionally, we analyzed net release times for only the two slowest speeds, and found a clear strategy to catch with the center of the net. This produced a significant effect of net size on net release times, $F(2,22)=3.91, p<.05, \eta_{\mathrm{p}}{ }^{2}=.26$ (big, $M=1,139 \mathrm{~ms}$; medium, $M=1,074 \mathrm{~ms}$; small, $M=1,039 \mathrm{~ms})$. Thus, for speeds at which the strategy was clearly to catch the fish with the center of the net, the critical effect still emerged.

Another factor that could have influenced net release times, and accounted for the proposed effects on perceived speed, was catching performance on the previous trial. If participants had released the net early on the previous trial, they may have adjusted their timing and been more likely to release the net later on the subsequent trial. However, whereas factors such as performance on previous trials did influence net release times, including these factors did not eliminate the critical effect of net size on net release times (see the supplementary materials). Regardless of prior performance, participants continued to release the big net later than the small net.

\section{Control experiment}

Another potential factor was that the fish was impossible to catch for certain speed/net size combinations, and the impossibility of the task under some conditions may have influenced performance under the other conditions. To examine whether this could account for our results, we conducted another experiment in which all of the fish could be caught. The design was the same as in the main experiment, except for the following changes: only the low fish and five fish speeds were used, the speeds were set to $3.2-$ $9.6 \mathrm{~cm} / \mathrm{s}$, and the participants $(N=12)$ estimated the speed of the fish by using a speed bisection task (Witt \& Sugovic, 2010, 2012, 2013).

Although net size still influenced fish-catching performance, $F>25, \eta_{\mathrm{p}}{ }^{2}>.69$, participants could catch the fish under all conditions (see the supplementary materials). Net size also influenced the explicit judgments of fish speed, $F(2,22)=4.28, p<.05, \eta_{\mathrm{p}}{ }^{2}=.28$ (see the supplementary materials). Critically, net size also influenced net release times, $F(2,22)=31.73, p<.001, \eta_{\mathrm{p}}{ }^{2}=.74$ : As in the original experiment, participants released the big net sooner than the small net, consistent with the idea that they perceived the fish to be moving more slowly when the net was big (see Fig. 5). This result demonstrates that even when all of the fish could be caught, net size still influenced net release times. Fish speed also influenced net release times, $F(4,44)>1,700$. The interaction was also significant, $F(8,88)=2.70, p=.01, \eta_{\mathrm{p}}{ }^{2}=.20$, but follow-up analyses showed that net size influenced net release times at all fish speeds, $p \mathrm{~s}<.05, \eta_{\mathrm{p}}{ }^{2} \mathrm{~s}>.32$.

\section{Discussion}

The action-specific account of perception posits that people see the world in a way that relates to their ability to perform the intended action (Proffitt, 2006; Witt, 2011a). The implication of this account is that the same object will look different, depending on the perceiver's abilities and intentions. In line with Gibson's (1979) ecological approach, the action-specific account emphasizes the idea that perception expresses the relationship between the perceiver and the environment.

However, much debate has concerned whether actionspecific effects reflect true changes in perception, or whether the changes occur instead in postperceptual processes. This debate has largely centered on methodological issues concerning measurements of perception. Perception cannot be measured directly, and instead must be inferred on the basis of judgments or behaviors. Thus, the locus of the effects could be in perception or in the processes that generate the responses.

Most of the empirical support for the action-specific account has come from explicit judgments of the target object, such as its distance, size, or speed. However, explicit judgments can be susceptible to factors such as response bias, task demands (e.g., Asch, 1955), and instructions (Carlson, 1977; Woods et al., 2009). Thus, a concern is whether a person's ability to act influences perceptions of the target, or whether manipulations of ability create a task demand that influences responses. Because of these concerns with using explicit judgments, the use of action-based measures has been suggested instead (e.g., Loomis \& Philbeck, 2008). Actionbased measures are unlikely to be influenced by factors such as instructions or task demands, because the instructions associated with action-based measures typically refer only to performance goals, and not to perception.

In the present experiment, a fish traveled across the screen, and participants pressed a trigger to shoot a net up to try to intercept the fish. We used net release time as an action-based measure of perceived fish speed. If the fish looked to be moving more slowly, participants should have waited longer to release the net. If the fish looked to be moving more quickly, they should have released the net sooner.

We found that participants waited longer to release the big net than to release the small net. This pattern is suggestive of participants seeing the fish as moving more slowly when playing with the big rather than the small net, and it corroborates those found with explicit judgments of fish speed. Parallel findings in both judgment-based and action-based measures provide converging evidence that task difficulty influences a common underlying process, such as perceived speed, rather than influencing responsespecific postperceptual processes (cf. Foley, 1977; Philbeck 
Fig. 5 Net release times, plotted as a function of net size and fish speed, for the control experiment, in which the fish could be caught in all conditions. The insert shows the main effect of net size collapsed across fish speeds. Error bars represent $1 S E M$ calculated within subjects

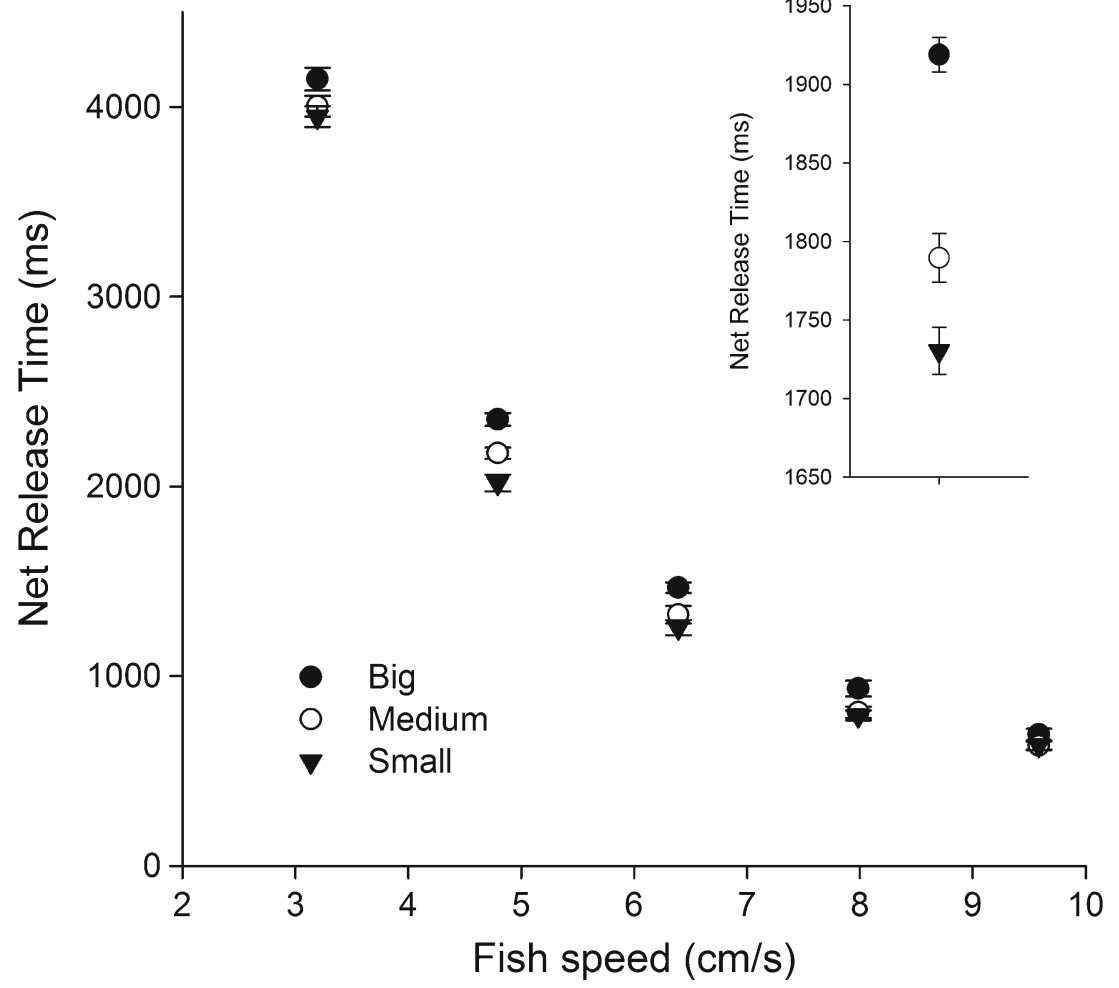

\& Loomis, 1997). Thus, for the first time, the present results provide direct support for the claim that catching difficulty influences perceived speed.

Given that catching ease influenced perceived fish speed, future research should also examine the underlying mechanism of this effect. One possibility is that these results are due to different allocations of attention: When the task is more difficult, perceivers might attend to the scene differently, which could lead to the fish's speed appearing to be faster. In this case, perception is still being influenced, but the mechanism involves attentional rather than perceptual processes. Despite some speculation (Bloesch, Davoli, Roth, Brockmole, \& Abrams, 2012), no work to date has examined the potential involvement of attention in these effects.

With respect to the consequences of these effects for behavioral decisions, there has only been speculation on the potentially adaptive nature of these effects (see the introduction). One possibility is that behaviorally dependent perceptions benefit actions. For example, seeing a golf hole as being bigger leads to enhanced putting performance (Witt, Linkenauger, \& Proffitt, 2012). Another possibility is that these effects exist out of necessity. The units of optical information are visual angles (see the figures in Proffitt \& Linkenauger, 2013). Other visual information-such as ocular-motor adjustments, retinal disparities, and changes in the angles of projection - also take the form of angles. Because the size of the projection in the eye of a given object depends on the distance to the object, these angles must be scaled in order to perceive features such as distance or size. It is possible that the only metrics available to scale this information are bodily and behaviorally based metrics such as eye height, arm length, and hand size (Linkenauger, Ramenzoni, \& Proffitt, 2010; Linkenauger, Witt, \& Proffitt, 2011; Proffitt \& Linkenauger, 2013), rather than behaviorally neutral metrics. Thus, perception may be action-specific because the optical information might be scaled by bodily and behaviorally based metrics.

Action-specific effects are consistent with a growing literature that has demonstrated an intimate connection between action-based and perceptual processes. For instance, the perception of biological motion and the perceived apparent motion of bodies are both influenced by the body's own repertoire of actions (Calvo-Merino, Glaser, Grèzes, Passingham, \& Haggard, 2005; Casile \& Giese, 2006; Oh \& Shiffrar, 2007; Shiffrar \& Freyd, 1990); the perception of object features such as orientation and direction is influenced by planned and executed movements (Lindemann \& Bekkering, 2009; Müsseler \& Hommel, 1997; Zwickel, Grosjean, \& Prinz, 2010); eye movements are drawn to objects oriented in the same direction as the planned grasp (Bekkering \& Neggers, 2002); and the detection of changes in orientation is enhanced when one is planning to grasp an object (Gutteling, Kenemans, \& Neggers, 2011). This body of research has demonstrated many influences of action on perception. 
In summary, the data show that difficulty associated with catching a virtual fish influences the perceived speed of the fish, as demonstrated by judgment-based and action-based measures. This converging evidence supports the idea that action-specific effects are perceptual, and it promotes the view that perceivers see the world in terms of their ability to act.

Authors Note This research was supported by the National Science Foundation (Grant No. BCS-0957051, to J.K.W.).

\section{References}

Asch, S. E. (1955). Opinions and social pressure. Scientific American, 193, 31-35. doi:10.1038/scientificamerican1155-31

Bekkering, H., \& Neggers, S. (2002). Visual search is modulated by action intentions. Psychological Science, 13, 370-374. doi:10.1111/j.0956-7976.2002.00466.x

Bhalla, M., \& Proffitt, D. R. (1999). Visual-motor recalibration in geographical slant perception. Journal of Experimental Psychology. Human Perception and Performance, 25, 10761096. doi:10.1037/0096-1523.25.4.1076

Bloesch, E. K., Davoli, C. C., Roth, N., Brockmole, J. R., \& Abrams, R. A. (2012). Watch this! Observed tool use affects perceived distance. Psychonomic Bulletin \& Review, 19, 177-183. doi:10.3758/ s13423-011-0200-Z

Calvo-Merino, B., Glaser, D. E., Grèzes, J., Passingham, R. E., \& Haggard, P. (2005). Action observation and acquired motor skills: An fMRI study with expert dancers. Cerebral Cortex, 15, 12431249. doi:10.1093/cercor/bhi007

Casile, A., \& Giese, M. A. (2006). Nonvisual motor training influences biological motion perception. Current Biology, 16, 69-74.

Cole, S., Balcetis, E., \& Dunning, D. (2013). Affective signals of threat produce perceived proximity. Psychological Science, 24, 34-40. doi: $10.1177 / 0956797612446953$

Carlson, V. R. (1977). Instructions and perceptual constancy judgments. In W. Epstein (Ed.), Stability and constancy in visual perception: Mechanisms and processes (pp. 217-254). New York: Wiley.

Durgin, F. H., Baird, J. A., Greenburg, M., Russell, R., Shaughnessy, K., \& Waymouth, S. (2009). Who is being deceived? The experimental demands of wearing a backpack. Psychonomic Bulletin \& Review, 16, 964-969. doi:10.3758/PBR.16.5.964

Foley, J. M. (1977). Effect of distance information and range on two indices of visually perceived distance. Perception, 6, 449-460.

Ganal, T., Tanzer, M., \& Goodale, M. A. (2008). A double dissociation between action and perception in the context of visual illusions opposite effects of real and illusory size. Psychological Science, 19, 221225 .

Gibson, J. J. (1979). The ecological approach to visual perception. Boston: Houghton Mifflin.

Gutteling, T. P., Kenemans, J. L., \& Neggers, S. F. W. (2011). Grasping preparation enhances orientation change detection. PLOS ONE, 6, e17675. doi:10.1371/journal.pone.0017675

Lee, Y., Lee, S., Carello, C., \& Turvey, M. T. (2012). An archer's perceived form scales the "hitableness" of archery targets. Journal of Experimental Psychology: Human Perception and Performance, 38, 1125-1131.

Lindemann, O., \& Bekkering, H. (2009). Object manipulation and motion perception: Evidence of an influence of action planning on visual processing. Journal of Experimental Psychology. Human Perception and Performance, 35, 1062-1071.
Linkenauger, S. A., Ramenconi, V. C., \& Proffitt, D. R. (2010). Illusory shrinkage and growth: Body-based rescaling affects the perception of size. Psychological Science, 21, 1318-1325.

Linkenauger, S. A., Witt, J. K., \& Proffitt, D. R. (2011). Taking a hands-on approach: Apparent grasping ability scales the perception of object size. Journal of Experimental Psychology. Human Perception and Performance, 37, 1432-1441. doi:10.1037/a0024248

Loomis, J. M., \& Philbeck, J. W. (2008). Measuring spatial perception with spatial updating and action. In R. L. Klatzky, B. MacWhinney, \& M. Behrmann (Eds.), Embodiment, ego-space, and action (pp. 1-44). New York: Psychology Press.

Müsseler, J., \& Hommel, B. (1997). Detecting and identifying response-compatible stimuli. Psychonomic Bulletin \& Review, 4, 125-129. doi:10.3758/BF03210785

Oh, S., \& Shiffrar, M. (2007). Apparent motion of the face [Abstract]. Journal of Vision, 7(9), 127. doi:10.1167/7.9.127

Philbeck, J. W., \& Loomis, J. M. (1997). Comparison of two indicators of visually perceived egocentric distance under full-cue and reduced-cue conditions. Journal of Experimental Psychology. Human Perception and Performance, 23, 72-85. doi:10.1037/0096-1523.23.1.72

Proffitt, D. R. (2006). Embodied perception and the economy of action. Perspectives on Psychological Science, 1, 110-122.

Proffitt, D. R., \& Linkenauger, S. A. (2013). Perception viewed as a phenotypic expression. In W. Prinz, M. Beisert, \& A. Herwig (Eds.), Action science: Foundations of an emerging discipline (pp. 171-198). Cambridge: MIT Press.

Shiffrar, M., \& Freyd, J. (1990). Apparent motion of the human body. Psychological Science, 1, 257-264.

Stefanucci, J. K., \& Proffitt, D. R. (2009). The roles of altitude and fear in the perception of height. Journal of Experimental Psychology. Human Perception and Performance, 35, 424-438. doi:10.1037/a0013894

Trommershäuser, J., Maloney, L. T., \& Landy, M. S. (2008). Decision making, movement planning and statistical decision theory. Trends in Cognitive Sciences, 12, 291-297. doi:10.1016/j.tics.2008.04.010

Witt, J. K. (2011a). Action's effect on perception. Current Directions in Psychological Science, 20, 201-206.

Witt, J. K. (2011b). Tool use influences perceived shape and parallelism: Indirect measures of perceived distance. Journal of Experimental Psychology. Human Perception and Performance, 37, 1148-1156.

Witt, J. K., Linkenauger, S. A., \& Proffitt, D. R. (2012). Get me out of this slump! Visual illusions improve sports performance. Psychological Science, 23, 397-399. doi:10.1177/0956797611428810

Witt, J. K., Proffitt, D. R., \& Epstein, W. (2010). When and how are spatial perceptions scaled? Journal of Experimental Psychology. Human Perception and Performance, 36, 1153-1160. doi:10.1037/a0019947

Witt, J. K., \& Sugovic, M. (2010). Performance and ease influence perceived speed. Perception, 39, 1341-1353.

Witt, J. K., \& Sugovic, M. (2012). Does ease to block a ball affect perceived ball speed? Examination of alternative hypotheses. Journal of Experimental Psychology. Human Perception and Performance, 38, 1202-1214. doi:10.1037/a0026512

Witt, J. K., \& Sugovic, M. (2013). Response bias cannot explain actionspecific effects: Evidence from compliant and non-compliant participants. Perception, 42, 138-152. doi:10.1068/p7367

Witt, J. K., \& Sugovic, M. (in press). Spiders appear to move faster than non-threatening objects regardless of one's ability to block them. Acta Psychologica.

Woods, A. J., Philbeck, J. W., \& Danoff, J. V. (2009). The various "perceptions" of distance: An alternative view of how effort affects distance judgments. Journal of Experimental Psychology: Human Perception and Performance, 35, 1104-1117. doi:10.1037/ a0013622

Zwickel, J., Grosjean, M., \& Prinz, W. (2010). On interference effects in concurrent perception and action. Psychological Research, 74, 152-171. doi:10.1007/s00426-009-0226-2 